\title{
EXTRATOS DE Lilium sp., Agapanthus sp. E Hydrangea sp.: COMPORTAMENTO COMO INDICADORES NATURAIS EM DIFERENTES FAIXAS DE pH
}

\author{
Jhulli M. da Silva ${ }^{\mathrm{a}}$, Renata de C. Martins ${ }^{\mathrm{a}}$, Fabiele Bernardia, Camila de M. R. dos Santos ${ }^{\mathrm{a}}$, Keller P. Nicolini ${ }^{\mathrm{a}}$ e Jaqueline \\ Nicolini*,a, (i) \\ aDepartamento de Química, Instituto Federal de Educação, Ciência e Tecnologia do Paraná, 85555-000 Palmas - PR, Brasil
}

Recebido em 27/06/2019; aceito em 07/10/2019; publicado na web em 10/01/2020

\begin{abstract}
EXTRACTS OF Lilium sp., Agapanthus sp. AND Hydrangea sp.: BEHAVIOR AS NATURAL INDICATORS IN DIFFERENT pH RANGE. This experimental activity proposes the use of lily (Lilium sp.), agapanthus (Agapanthus sp.) and hydrangea (Hydrangea sp.) extracts to investigate the colorimetric profile of each extract between $\mathrm{pH} 1-14$. Water, ethanol and ethanol/HCl $1 \%$ (w/w) were used as extractive solvents. Among the flowers tested, the extracts of orange lily and burnt yellow lily were the ones that presented the greatest color variation with the solution $\mathrm{pH}$ change. In addition, the total content of anthocyanins in relation to cyanidin-3-glycosidic was determined and in ascending order we have: yellow lily (LAM: $0,07 \pm 0,03$ ) < agapanthus white (AGB: $0,21 \pm 0,03)<$ agapanthus violet (AGV: $1,72 \pm 0,13$ ) < blue hydrangea (HAZ: 1,83 $\pm 0,47)<$ orange lily (LAL: $2,87 \pm 0,52$ ) < burnt yellow lily (LAQ: $9,55 \pm 0,46)$. For this determination methanol/HCl $1 \%(\mathrm{w} / \mathrm{w})$ was used as the solvent. Using UV-Vis spectroscopy, it is suggested that the behaviors are due to the presence of cyanidin in AGB, AGV, LAL and LAQ, delphinidin in HAZ and apigeninidine in LAM. This activity can be applied in courses that have Chemistry in their curricular matrix and in General Chemistry subjects when the acid-base theme is approached.
\end{abstract}

Keywords: natural pH indicator; anthocyanins; experiment proposal; acid-base.

\section{INTRODUÇÃO}

Os conceitos de ácido e de base são multidisciplinares, pois eles podem ser abordados em diferentes disciplinas de uma mesma área ou de diferentes áreas, por exemplo, em química, bioquímica, geologia ou biologia, mantendo a integridade do conceito. O conceito ácido-base pode ser empregado quando se pretende determinar o $\mathrm{pH}$ do solo ${ }^{1}$ ou a presença de ácidos húmicos no solo, ${ }^{2}$ em sistemas coloidais, ${ }^{3} \mathrm{em}$ sistemas supramoleculares proteicos como a hemoglobina, ${ }^{4} \mathrm{em}$ estudos de equilíbrio químico ${ }^{5,6}$ ou em volumetria de neutralização, ${ }^{7,8}$ por exemplo. Sumariamente, a Tabela 1 apresenta as definições clássicas dos conceitos de ácido e de base: ${ }^{9}$

Tabela 1. Definição da literatura9,10 para os conceitos de ácido e de base

\begin{tabular}{lll}
\hline & \multicolumn{1}{c}{ Ácido } & \multicolumn{1}{c}{ Base } \\
\hline \multirow{2}{*}{ Arrhenius } & $\begin{array}{l}\text { Composto que, em } \\
\text { solução aquosa, produz } \\
\text { íons de hidrogênio }\left(\mathrm{H}^{+}\right)\end{array}$ & $\begin{array}{l}\text { Composto que, em solução } \\
\text { aquosa, produz íons de } \\
\text { hidroxila }(\mathrm{OH})\end{array}$ \\
\hline Brönsted-Lowry & $\begin{array}{l}\text { Espécie que age como } \\
\text { doador de prótons }\left(\mathrm{H}^{+}\right)\end{array}$ & $\begin{array}{l}\text { Espécie que age como re- } \\
\text { ceptor de prótons }\left(\mathrm{H}^{+}\right)\end{array}$ \\
\hline Lewis & $\begin{array}{l}\text { Espécie que age como um } \\
\text { receptor de par eletrônico }\end{array}$ & $\begin{array}{l}\text { Espécie que age como um } \\
\text { doador de par eletrônico }\end{array}$ \\
\hline
\end{tabular}

$\mathrm{O}$ pHé um dos fatores mais importantes para definir a pigmentação das flores ou frutos, pois a acidez do vacúolo celular varia entre 2,5 e 7,5 sobre as antocianinas. De acordo com Brouillard, ${ }^{11}$ entre $\mathrm{pH} 1 \mathrm{e}$ 2 o cátion flavínico $\left(\mathrm{AH}^{+}\right)$é provavelmente o único responsável pela pigmentação enquanto entre $\mathrm{pH}$ 3-4 tanto o cátion flavínico como as bases quinoidais neutras (tautômeros neutros) contribuem para a cor. Já entre pH 4-6 as bases quinoidais neutras são majoritariamente as responsáveis pela coloração observada, enquanto em pH 7 a espécie carregada negativamente é tão estável quanto a base quinoidal. As bases

*e-mail: jaqueline.nicolini@ifpr.edu.br quinoidais neutras (A) ou negativamente carregadas (A') apresentam estabilidade em $\mathrm{pH}$ 10. Nas soluções aquosas ácidas existem quatro espécies de antocianinas em equilíbrio: ${ }^{11} \mathrm{i}$ ) a base quinoidal (A ou $\mathrm{A}^{-}$); ii) o cátion flavínico $\left(\mathrm{AH}^{+}\right)$; iii) a pseudobase ou carbinol (B); e iv) a chalcona $(\mathrm{C})$. A representação esquemática da interconversão entre essas quatro estruturas é apresentada no Esquema 1. As antocianinas são corantes naturais ${ }^{12}$ moderadamente hidrossolúveis, estáveis em meio ácido, encontradas nos vacúolos de muitas plantas. ${ }^{13}$ Elas são responsáveis pela cor vermelho à azul de muitas flores, frutos e legumes. ${ }^{14}$ Há muitas antocianinas já identificadas na natureza devido ao número de variações possíveis com mono- ou diglicosídeos e de mono-, di- ou trissacarídeos. As antocianinas encontram-se associadas a moléculas de açúcares e, quando livres desses açúcares, são denominadas antocianidinas (agliconas), ${ }^{15}, 16$ que além de atuarem como antioxidantes e agentes quelantes ainda se interconvertem (Esquema 1) alterando a coloração do meio em função da alteração de $\mathrm{pH} \cdot{ }^{17}$ Algumas antocianidinas são apresentadas na Figura 1.

\section{OBJETIVOS}

O Brasil é um país de dimensão continental e isso reflete em uma grande variedade da flora que se encontra distribuída pelos diferentes biomas brasileiros. Uma grande variedade de frutas e vegetais contêm esse grupo de flavonoides conhecidos como antocianinas, os quais são responsáveis pela cor azul-vermelho observadas em flores e frutos. ${ }^{22}$ A diversidade da flora brasileira vem contribuindo para que diversos pesquisadores possam construir atividades experimentais, ${ }^{7,23-41}$ compondo uma espécie de "coleção de atividades", que retratam as diversas potencialidades da flora que cada região do Brasil possui, vistas as diferentes realidades escolares do Brasil. O método proposto amplia a flora que pode ser utilizada como indicador natural de $\mathrm{pH}$, apresentando estabilidade da cor, facilidade de obtenção e utilização de reagentes acessíveis, despertando um olhar investigativo sobre o tema. Dessa forma, esta atividade visa propor estratégias que minimizem a geração de produtos que apresentam toxicidade para a saúde 


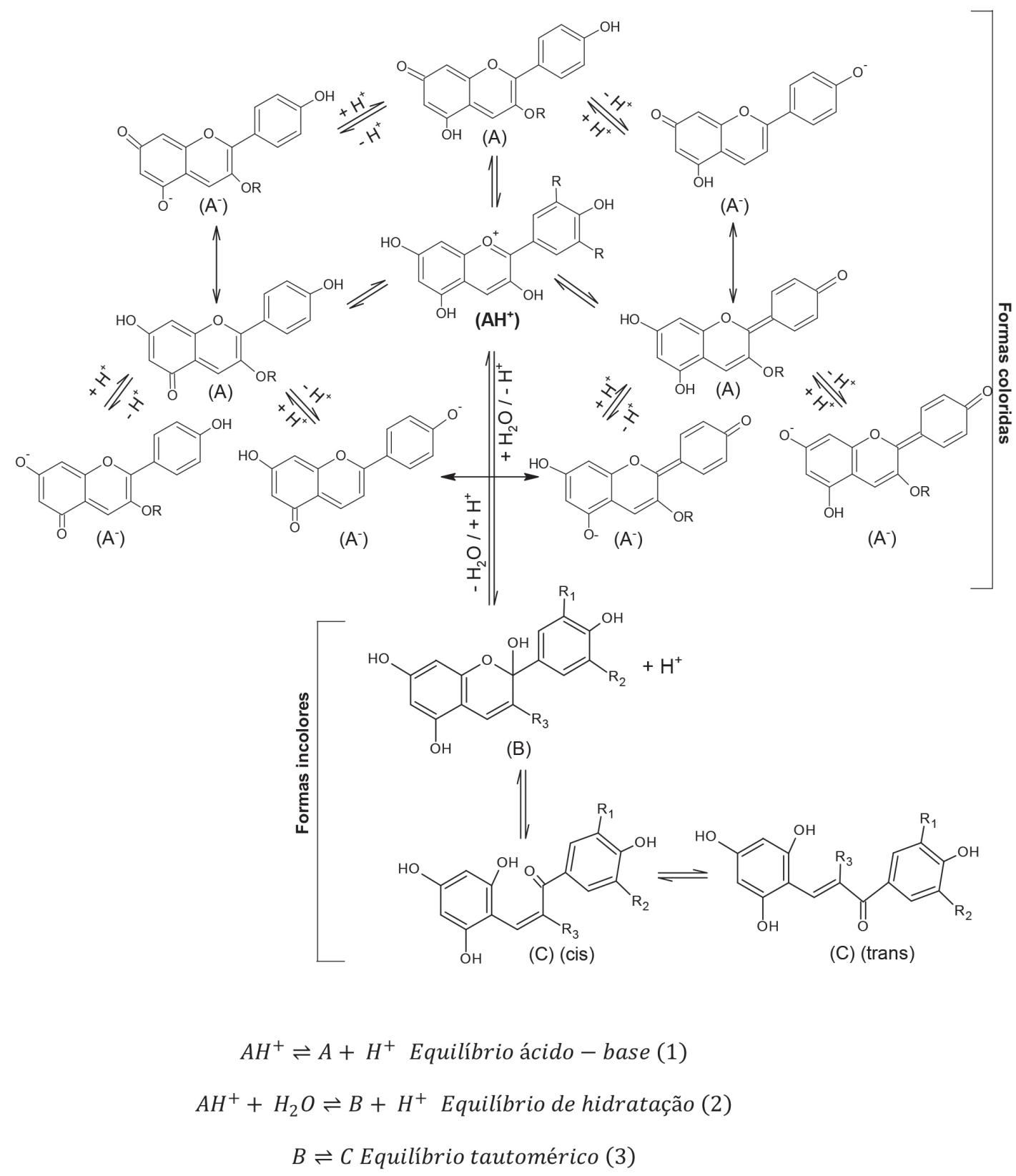

Esquema 1. Interconversão entre as estruturas de equilíbrio das antocianinas ${ }^{11,18-20}$

dos estudantes e para o ambiente, sendo esse um dos 12 princípios da química verde. Esta atividade experimental tem como objetivos:

- Utilizar extratos de flores para abordar o tema ácido-base de forma multidisciplinar.

- Obter uma tabela colorimétrica utilizando os extratos de flores como indicadores naturais de $\mathrm{pH}$ valorizando as potencialidades da flora de cada região do Brasil.

- Determinar o teor de antocianinas presentes nas flores testadas.

- Discutir sobre as plantas mais adequadas para este tipo de atividade, bem como o solvente mais indicado, visando menor gasto com reagentes e uso de plantas que apresentem as maiores variações colorimétricas frente à mudança de $\mathrm{pH}$.

\section{PARTE EXPERIMENTAL}

Para o cálculo do teor de antocianinas em relação à cianidina-3-glicosídica, utilizou-se metanol acidulado com $\mathrm{HCl}$ a $1 \%$ como solvente extrator. ${ }^{42}$ Em seguida, para investigar o comportamento dos extratos de Lilium, Agapanthus e Hydrangea em diferentes faixas de $\mathrm{pH}$, dois métodos de extração foram empregados, visando determinar o método e a espécie de planta mais adequada para a obtenção do extrato vegetal e consequentemente das tabelas colorimétricas, que indicam o comportamento de cada extrato entre $\mathrm{pH} 1-14$. Método $1^{7}$ - utilizando etanol e água e Método $2^{16}$ - utilizando etanol acidulado com $\mathrm{HCl}$ a $1 \%$.

\section{Materiais, reagentes e equipamentos}

Os reagentes e os solventes utilizados foram de grau analítico: etanol (Neon, 99,5\%), metanol (Neon, 99,8\%), hidróxido de sódio (Neon, 99,0\%) ácido clorídrico (Vetec, 37,0\%), água destilada ( $<10 \mu \mathrm{S} \mathrm{cm}^{-1}$ ), pétalas de (a) lírio (Lilium sp.) nas cores alaranjado (LAL), amarelo-queimado (LAQ) e amarelo (LAM); (b) agapanto (Agapanthus sp.) nas cores branco (AGB) e violeta (AGV); (c) hortensia (Hydrangea sp.) azul (HAZ), pHmetro digital de bancada (pHS-38W microprocessor) utilizando soluções tampão (Vetec) 


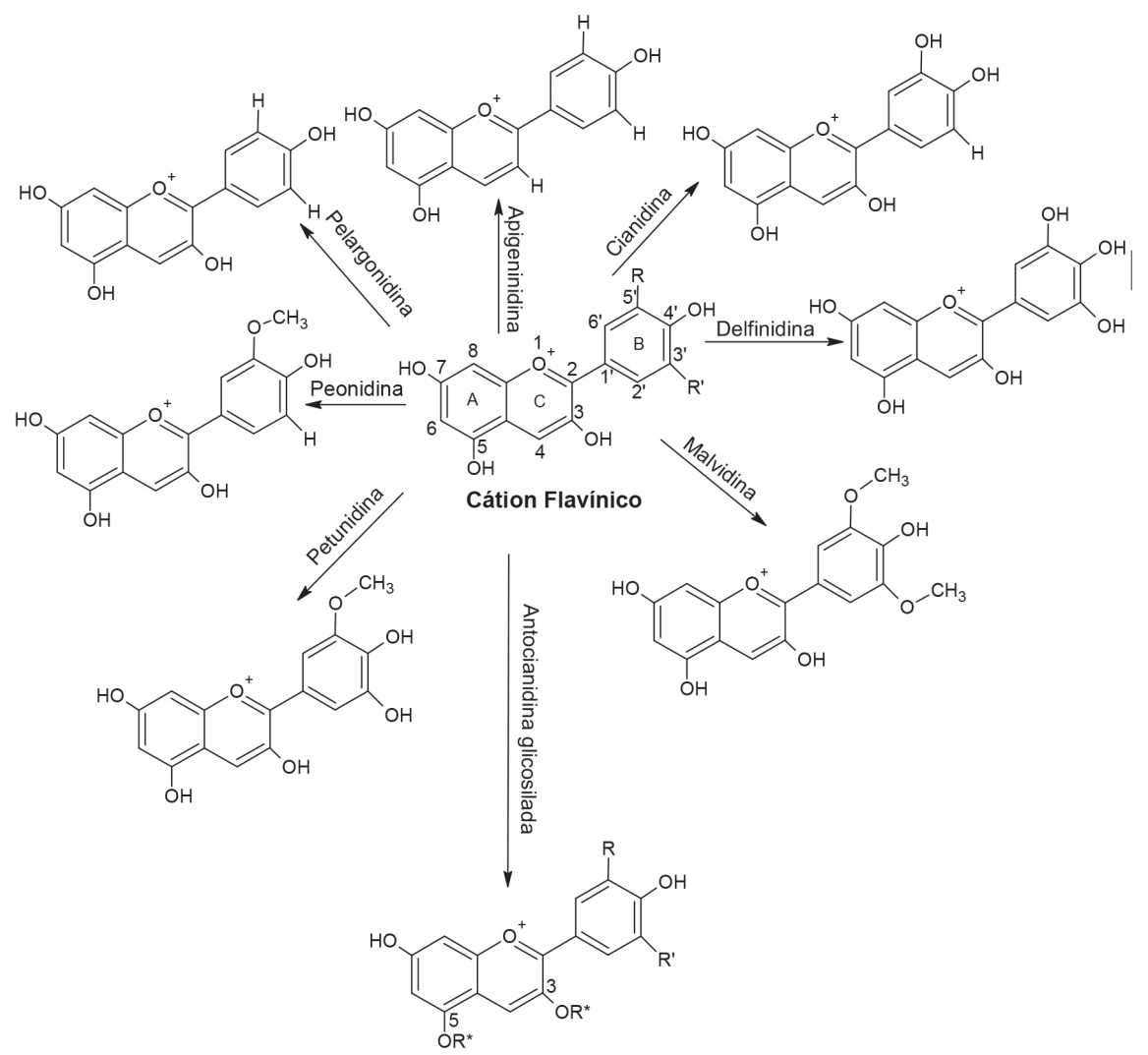

Figura 1. Representação de algumas antocianidinas. Sendo $R^{*}$ uma molécula de açúcar ${ }^{16,21}$

pH 4,00 e pH 7,00 para calibrar o pHmetro, espectrofotometro PerkinElmer Lambda 365. As flores foram coletadas no jardim do campus da instituição. A Figura 2 apresenta a imagem das flores utilizadas para os testes realizados.

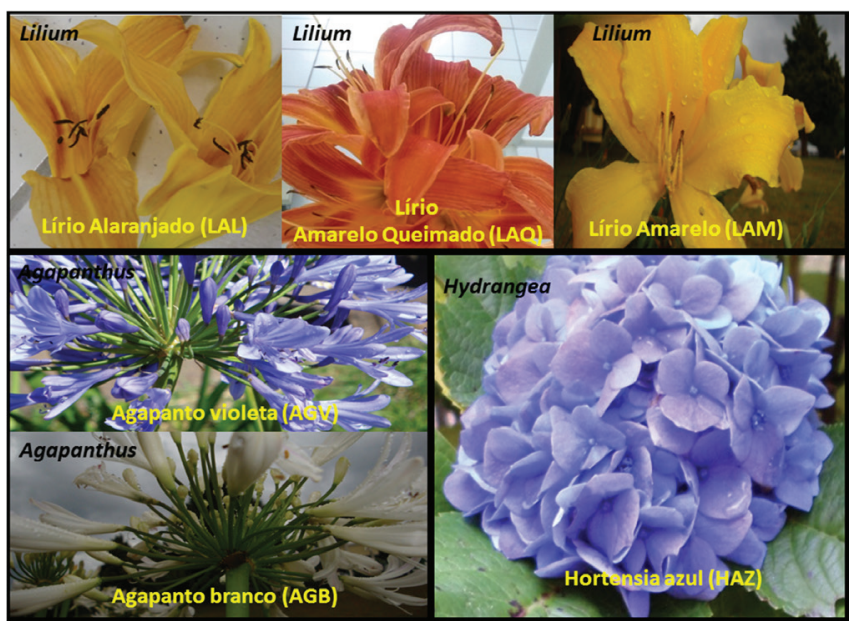

Figura 2. Imagem das flores estudadas, nomes comuns (em amarelo) $e$ científicos (em itálico)

\section{Método para a determinação do teor de antocianinas}

O teor de antocianinas (TA) é calculado em porcentagem (\%) em relação à cianidina-3-glicosídica ${ }^{42}$ utilizando a Equação 1 , a qual é aplicada quando o solvente utilizado é $\mathrm{MeOH} / \mathrm{HCl}, 1 \%,{ }^{42}$ sendo A o valor da absorbância medida em $528 \mathrm{~nm}$, FD o fator de diluição, 772 a absorbância específica da cianidina-3-glicosídica e m a massa da $\operatorname{amostra}(\mathrm{g})$. A extração foi realizada utilizando $0,1000 \pm 0,0030 \mathrm{~g}$ de material vegetal e $5,0 \mathrm{~mL}$ de metanol acidulado com $\mathrm{HCl}$ a $1 \%$
$(\mathrm{MeOH} / \mathrm{HCl}, 1 \%)$. Esse método permite a extração completa de antocianinas. ${ }^{16} \mathrm{O}$ extrato foi filtrado e ajustado para $10 \mathrm{~mL}$ com $\mathrm{MeOH} / \mathrm{HCl}, 1 \%$ (w/w). As extrações foram realizadas em triplicata, isto é, para três conjuntos de amostras de cada flor. Para cada conjunto de amostras foi determinado o teor de antocianinas de acordo com o método descrito por Sandre e col., ${ }^{42}$ o qual utiliza $\mathrm{MeOH} / \mathrm{HCl}, 1 \%$. A absorbância foi coletada logo após a extração para minimizar os efeitos de fotodegradação.

$$
T A=\left[\frac{(A \times F D}{(772 \times m}\right] \times 100
$$

\section{Métodos para a obtenção dos extratos vegetais e das tabelas colorimétricas}

Após o período de extração, utilizando o Método 1 e o Método 2 abaixo descritos, com o auxílio de uma pipeta de Pasteur, os extratos foram coletados e as tabelas colorimétricas foram obtidas nas diferentes faixas de $\mathrm{pH}$.

\section{Extração através do método 1}

Foram utilizados $5,0000 \pm 0,0010 \mathrm{~g}$ de pétalas recém-colhidas em $5,0 \mathrm{~mL}$ de solvente (água destilada e etanol 99,5\%), separadamente. As pétalas foram maceradas e transferidas para um tubo de ensaio com o auxílio de um bastão de vidro, e foram mantidas imersas, em cada um dos solventes, durante 48 horas $^{7}$ ao abrigo do calor e da luz. Essa extração ocorreu à $25^{\circ} \mathrm{C}$. Através desse método são extraídas majoritariamente antocianinas, apesar do etanol em concentrações superiores a $60 \%$ ser considerado adequado para obtenção de antocianidinas (agliconas). ${ }^{43}$

Extração através do método 2

$\mathrm{O}$ método para extração de antocianinas recomenda o uso de 
metanol ou etanol acidulado com $\mathrm{HCl}$ a $1 \%$ (w/w). Visando uma atividade que possa ser realizada em diferentes realidades escolares e que minimize a geração de produtos que apresentam toxicidade para a saúde dos estudantes e para o ambiente, foi utilizado etanol acidulado com $\mathrm{HCl}$ a $1 \%(\mathrm{EtOH} / \mathrm{HCl}, 1 \%$, w/w). As pétalas foram maceradas e transferidas para um tubo de ensaio com o auxílio de um bastão de vidro e mantidas imersas no solvente extrator durante 40 min a $80{ }^{\circ} \mathrm{C} .{ }^{16}$ Para isso, foi utilizado $1,0000 \pm 0,0010 \mathrm{~g}$ de pétalas recém-colhidas em 5,0 $\mathrm{mL}$ de solvente $(\mathrm{EtOH} / \mathrm{HCl}, 1 \%)$. Esse é um procedimento para pequenas quantidades de tecidos vegetais, para extração de antocianinas na forma livre de açúcares, as antocianidinas. ${ }^{16}$

Obtenção de tabela colorimétrica a partir do comportamento dos extratos em diferentes faixas de $\mathrm{pH}$

Para a obtenção da tabela colorimétrica foi preparada uma solução de $\mathrm{HCl}$ na concentração de $0,1 \mathrm{~mol} \mathrm{~L}^{-1}$ e uma solução de $\mathrm{NaOH}$ na concentração de $1,0 \mathrm{~mol} \mathrm{~L}^{-1}$. O valor do $\mathrm{pH}$ de cada solução foi medido com um pHmetro digital de bancada após calibração com as soluções tampão em pH 4,00 e em pH 7,00, com erro de $\pm 0,02$. A solução de $\mathrm{HCl} 0,1 \mathrm{~mol} \mathrm{~L}^{-1}$ apresentou pH 1,02 e a solução de $\mathrm{NaOH} 1,0 \mathrm{~mol} \mathrm{~L}^{-1}$ apresentou $\mathrm{pH} 14,00$. As demais soluções foram preparadas misturando-se as soluções de $\mathrm{HCl}$ e $\mathrm{NaOH}$ até obter soluções nos valores de $\mathrm{pH}: 2,02 ; 3,02 ; 4,03 ; 5,03 ; 6,02 ; 7,00 ; 8,03$; 9,$02 ; 10,01 ; 11,02 ; 12,02$ e 13,00. Em seguida, separou-se $1,0 \mathrm{~mL}$ de cada solução preparada e a elas foram adicionadas 4 gotas de cada extrato obtido, através do método 1 e do método 2 . Após a adição da solução indicadora preparada com $\mathrm{EtOH} / \mathrm{HCl}, 1 \%$ verificou-se variação de $\mathrm{pH}$ de $\pm 0,1$. Ao todo, foram preparadas 14 soluções entre $\mathrm{pH} 1-14$, e com elas obtiveram-se 18 tabelas colorimétricas provenientes do comportamento dos 18 extratos obtidos nas diferentes faixas de $\mathrm{pH}$.

\section{Análise por espectroscopia na região do visível}

Foi realizada a análise por espectroscopia na região do visível para os extratos obtidos através do método 2. Esses extratos não apresentaram turbidez, sendo viáveis para a coleta dos espectros, além de estarem de acordo com o método clássico de identificação de antocianinas, descrito por Harborne, ${ }^{21}$ o qual descreve que é possível utilizar etanol acidulado com $\mathrm{HCl}$ a $1 \%(\mathrm{EtOH} / \mathrm{HCl}, 1 \%)$ para identificar a região que as antocianidinas apresentam banda de absorção $\left(\lambda_{\max }\right)$ na região visível do espectro. ${ }^{21}$ Para as análises por espectroscopia na região do visível, foi utilizada cubeta de quartzo em um espectrofotômetro PerkinElmer Lambda 365, entre 400 e $700 \mathrm{~nm}$. Os espectros foram coletados logo após a extração para minimizar efeitos de fotodegradação. O uso de $\mathrm{EtOH} / \mathrm{HCl} 1 \%$ como solvente para a obtenção dos espectros visa o emprego de solventes que minimizem os impactos ambientais ou risco ao usuário, contemplando os princípios de Química Verde ou de Química Sustentável.

\section{Público alvo}

Essa estratégia pode ser aplicada em cursos de Química (Licenciatura ou Bacharelado), em disciplinas de Química Geral Experimental ou, ainda, em cursos como Farmácia e Biologia ou outros que tenham disciplinas de Química Geral. A Figura 3 apresenta de forma esquemática os procedimentos realizados neste manuscrito.

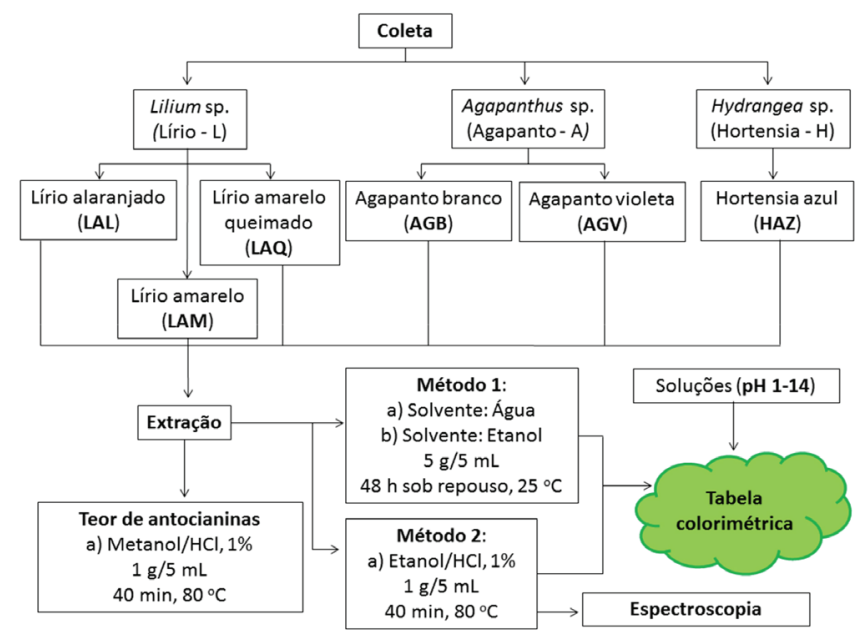

Figura 3. Representação esquemática do procedimento realizado

\section{RESULTADOS E DISCUSSÃO}

O teor de antocianinas foi determinado a partir das flores de Lilium sp., Agapanthus sp. e Hydrangea sp. utilizando a Equação 1 e indica a porcentagem de antocianinas em relação à cianidina-3-glicosídica. O lírio amarelo queimado (LAQ) foi a amostra que apresentou o maior teor de antocianinas, enquanto o lírio amarelo (LAM) apresentou o menor teor de antocianinas, conforme apresentado na Tabela 2, sendo que em ordem crescente temos: $\mathrm{LAM}<\mathrm{AGB}<\mathrm{AGV}<\mathrm{HAZ}<\mathrm{LAL}<\mathrm{LAQ}$.

Sabe-se que entre vegetais, flores e frutos as colorações de vermelho a violeta são derivadas principalmente de uma classe de flavonoides denominada antocianinas..$^{44} \mathrm{~A}$ partir da Tabela 2 observa-se que dentre as flores testadas, as amarelas e as brancas são as que apresentaram os menores teores de antocianinas. As flores investigadas com colorações mais próximas ao vermelho apresentaram os maiores teores de antocianinas (LAQ e LAL) e as flores violetas e azuis (AGV e HAZ) apresentaram teores intermediários de antocianinas. Existem principalmente três antocianinas que estão presentes em plantas superiores: pelargonidina, cianidina ou delfinidina e seus derivados, no entanto, muitas espécies de plantas acumulam flavonóis ou flavonas, além de antocianinas..$^{45} \mathrm{~A}$ literatura descreve que diferentes espécies vegetais apresentam diferentes teores de antocianinas e que a utilização da espectroscopia de UV-Vis na análise de extratos permite que os estudantes realizem a interpretação de resultados com precisão chegando a conclusões razoáveis. ${ }^{16,21,22,46,47}$

As antocianinas são responsáveis pela alteração de coloração do meio mediante alteração do $\mathrm{pH}$ da solução. ${ }^{7,25,26,48}$ Considerando-se os valores de absorbância máxima $\left(\lambda_{\max }\right)$, os resultados obtidos neste trabalho são coerentes com o trabalho de Harborne ${ }^{21}$ na identificação de cianidina, nesse caso presente nos extratos de LAQ, LAL, AGV e AGB; de delfinidina, presente no extrato de HAZ, e apigeninidina, no extrato de LAM (Tabela 3, Figura 4). As antocianidinas têm absorbância na região do ultravioleta (UV) e no visível do espectro (Vis), mas a intensidade de absorção no ultravioleta também é atribuível a outros compostos presentes no extrato, dessa forma, os espectros foram coletados entre 400 e $700 \mathrm{~nm} .^{22}$

Para os extratos de LAL e LAM, os resultados obtidos por espectroscopia na região do visível (Figura 4) indicam que essas flores

Tabela 2. Teor de antocianinas determinado nas flores das espécies investigadas, determinado por espectroscopia na região do visível

\begin{tabular}{|c|c|c|c|c|c|c|}
\hline Amostra & LAM & AGB & AGV & HAZ & LAL & LAQ \\
\hline $\begin{array}{l}\text { Teor de antocianina \% } \\
( \pm \text { Desvio Padrão) }\end{array}$ & $\begin{array}{c}0,07 \\
( \pm 0,03)\end{array}$ & $\begin{array}{c}0,21 \\
( \pm 0,03)\end{array}$ & $\begin{array}{c}1,72 \\
( \pm 0,13)\end{array}$ & $\begin{array}{c}1,83 \\
( \pm 0,47)\end{array}$ & $\begin{array}{c}2,87 \\
( \pm 0,52)\end{array}$ & $\begin{array}{c}9,55 \\
( \pm 0,46)\end{array}$ \\
\hline
\end{tabular}


sejam ricas em compostos da classe dos carotenoides ${ }^{49}$ tais como luteína e $\beta$-caroteno, presente em extratos de lírios. ${ }^{45} \mathrm{O} \beta$-caroteno apresenta três bandas de absorção características na região de 417 , $450,478 \mathrm{~nm}^{46}$ e a luteína na região de 421,445 e $474 \mathrm{~nm},{ }^{47}$ muito próximas às regiões observadas neste trabalho para os extratos de LAM (em 422, 447 e 473 nm) e LAL (em 422, 447 e 479 nm).

Tabela 3. Valores de $\lambda_{\max }$ obtidos através de espectroscopia na região do visível para os corantes contidos nos extratos investigados

\begin{tabular}{cccc}
\hline \multirow{2}{*}{ Extrato } & \multicolumn{2}{c}{$\lambda_{\max }(\mathrm{nm})$ em EtOH/HCl, 1\% } & \\
\cline { 2 - 3 } & Este trabalho & Ref. $^{21}$ & \\
\hline AGB & 549 & 545 & Cianidina \\
AGV & 549 & 545 & Cianidina \\
HAZ & 556 & 557 & Delfinidina \\
LAL & 544 & 545 & Cianidina \\
LAM & 473 & 483 & Apigeninidina \\
LAQ & 549 & 545 & Cianidina \\
\hline
\end{tabular}

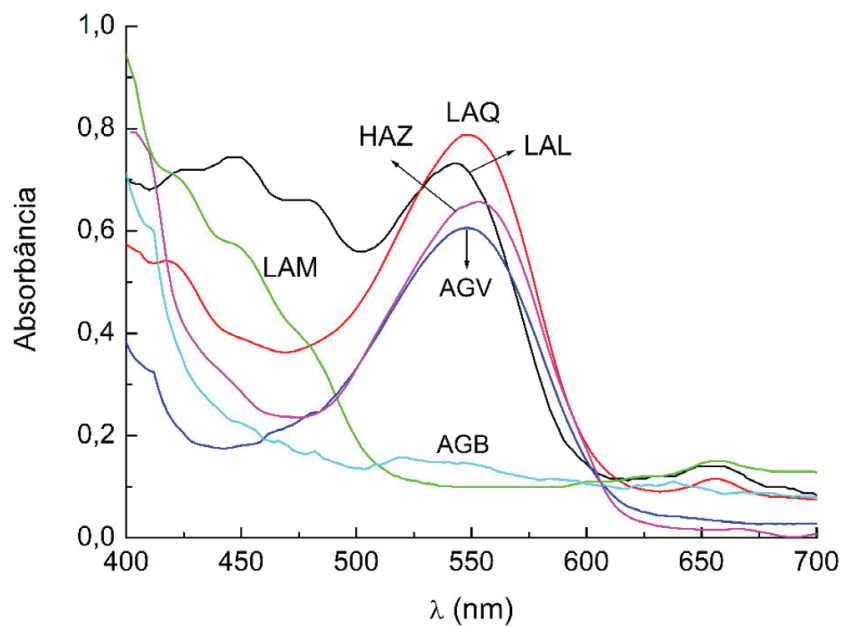

Figura 4. Espectros na região do visível obtidos a partir dos extratos de AGB (一), AGV (一), HAZ (一), LAL (一), LAM(—) e LAQ (一) em EtOH/HCl, $1 \%$

Entre as Figuras 5-10 são apresentadas as tabelas colorimétricas obtidas a partir das extrações realizadas. Em todas as figuras, o frasco à esquerda apresenta as colorações dos extratos das pétalas de cada espécie de flor em cada solvente e o pH das soluções está indicado em cada frasco em ordem crescente, da esquerda para a direita.

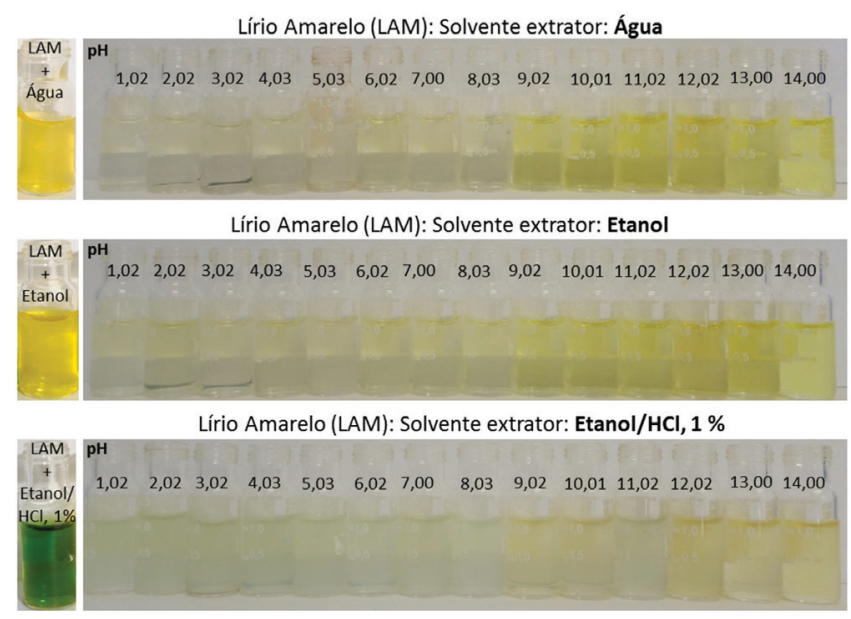

Figura 5. Tabela colorimétrica dos extratos de LAM

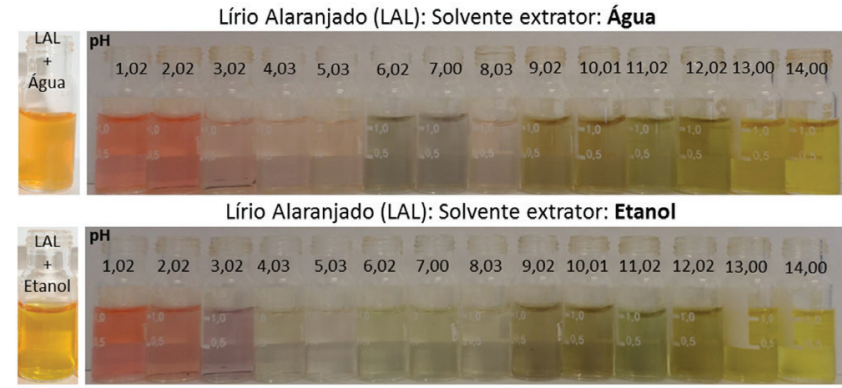

Lírio Alaranjado (LAL): Solvente extrator: Etanol/HCl, $1 \%$

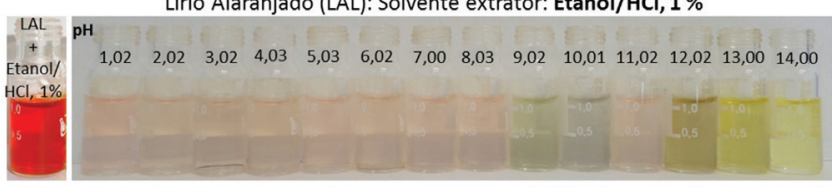

Figura 6. Tabela colorimétrica dos extratos de LAL
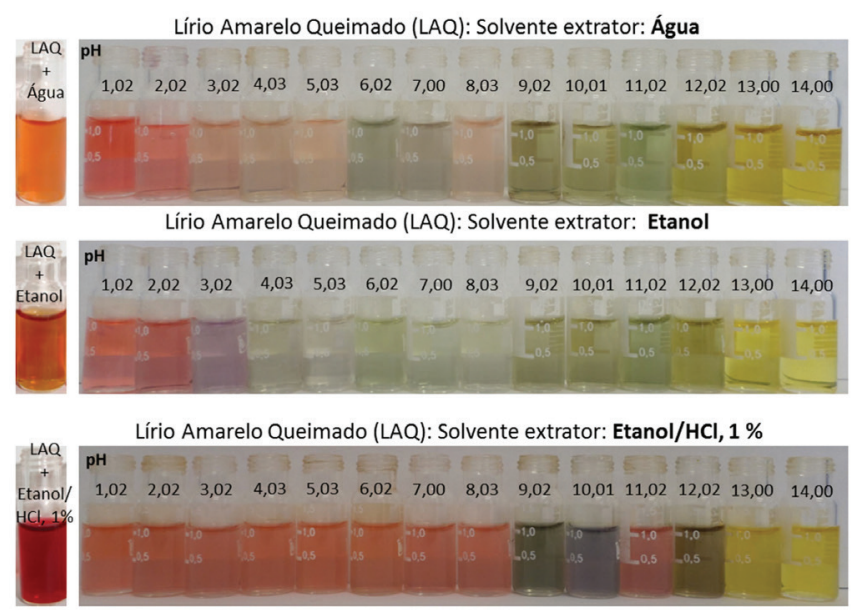

Figura 7. Tabela colorimétrica dos extratos de LAQ

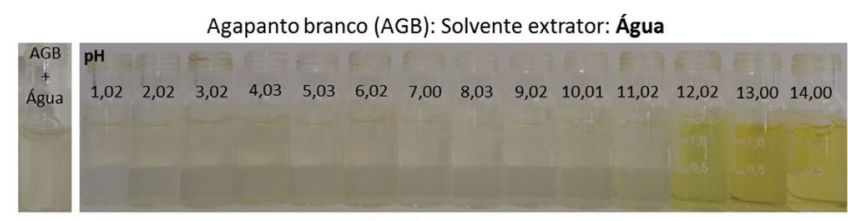

Agapanto branco (AGB): Solvente extrator: Etanol

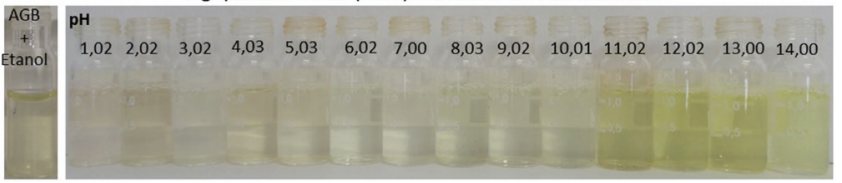

Agapanto branco (AGB): Solvente extrator: Etanol/HCl, $1 \%$

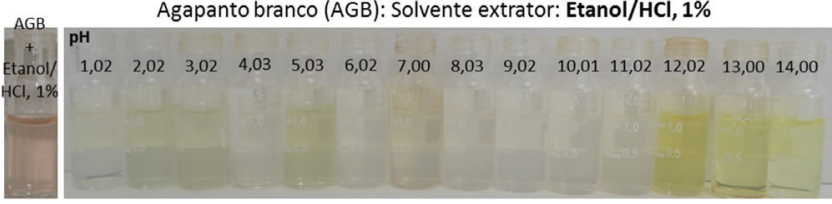

Figura 8. Tabela colorimétrica dos extratos de AGB

A Figura 5 (extratos de LAM) e a Figura 8 (extratos de AGB) apresentam as tabelas colorimétricas com as menores variações e intensidades de cor, o que se justifica pelos baixos teores de antocianinas calculados para estas flores (Tabela 2).

A Figura 6, referente aos extratos de LAL, a Figura 7, referente aos extratos de LAQ, e a Figura 9, referente aos extratos de AGV, apresentam as maiores variações colorimétricas. Os extratos de LAL e LAQ são os que apresentam as cores mais intensas pelo maior teor 

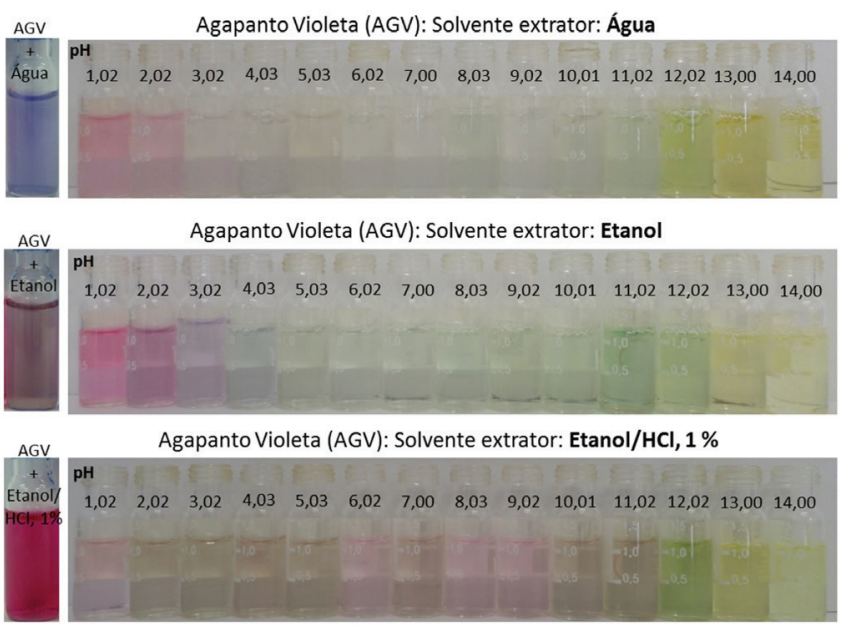

Figura 9. Tabela colorimétrica dos extratos de AGV

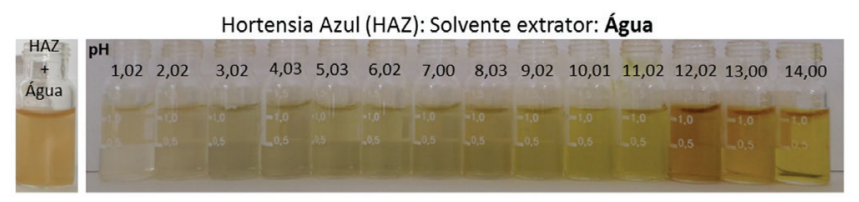

Hortensia Azul (HAZ): Solvente extrator: Etanol

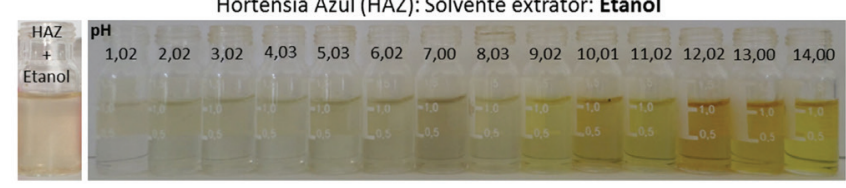

Hortensia Azul (HAZ): Solvente extrator: Etanol/HCl, 1\%

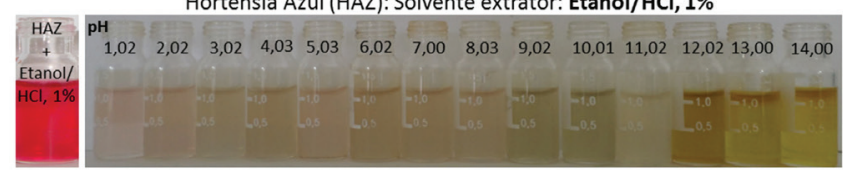

Figura 10. Tabela colorimétrica dos extratos de HAZ

antocianinas (Tabela 2). Essa sequência colorimétrica é semelhante ao comportamento de extratos de repolho roxo ${ }^{26,50}$ e de extratos de feijão-preto, ${ }^{7,25}$ situações em que se monitorou a coloração dos extratos utilizando produtos do cotidiano dos estudantes. Estudos descritos na literatura demonstraram que extratos de feijão-preto contêm delfinidina e pelargonidina, ${ }^{23}$ o que permite a aplicação de extratos brutos da casca de feijão-preto em estudos envolvendo volumetria ácido-base. ${ }^{7}$ Lírios geralmente possuem as antocianinas cianidina, pelargodinina, delfinidina e seus derivados, ${ }^{45,51}$ assim como o repolho-roxo e o feijão-preto. ${ }^{52}$ Nesta atividade experimental, o comportamento observado se deve à cianidina em LAL e em LAQ (Tabela 3, Figura 4), sendo o corante responsável pela diversidade de cores nas tabelas colorimétricas apresentadas para LAL (Figura 7) e para LAQ (Figura 9).

No extrato de AGV (Figura 9), o perfil da tabela colorimétrica obtida pode ser atribuída à presença das antocianinas geralmente presentes nessa espécie, sendo elas a delfinididina, petunidida e cianidina e/ou derivados glicosilados. ${ }^{53-55}$ A presença da banda de absorção em $549 \mathrm{~nm}$ para o extrato de AGV verificada por espectroscopia na região do visível (Figura 4, Tabela 3) indica que o comportamento observado na tabela colorimétrica (Figura 9) se deve à presença de cianidina (Tabela 3, Figura 4). Os extratos de LAM (Figura 5) não seguem esse perfil, pois as variações colorimétricas vão de incolor (até $\mathrm{pH} 8,03$ ) a amarelo (até pH 14,00). Essa espécie de lírio apresenta baixo teor de antocianinas e os carotenoides $\beta$-caroteno e/ou luteína (Figura 11), fato evidenciado pelas três bandas de absorção características de $\beta$-caroteno na região de 417, 450, $478 \mathrm{~nm}^{46}$ ou de luteína na região de 421, 445 e 474 nm. ${ }^{47}$ Carotenoides são corantes naturais presentes em flores e frutas e têm como função atrair polinizadores e agentes de dispersão de sementes. ${ }^{45}$ Os extratos de HAZ (Figura 10) geralmente apresentam delfinidina, cianidina e seus derivados. ${ }^{56,57}$ Nesse caso, é provável a presença de delfinidina, em função da presença da banda de absorção em $556 \mathrm{~nm}$ verificada por espectroscopia na região do visível (Figura 4, Tabela 3). As soluções na presença do extrato de HAZ apresentam colorações que vão do róseo ao amarelo, passando pelo verde (entre pH 9,02 e 10,01). Observa-se que a intensidade das cores obtidas é menor do que às obtidas a partir dos extratos de LAL e LAQ, provavelmente pelo menor teor de antocianinas presentes em HAZ (de 1,82\%), quando comparadas ao teor de $2,87 \%$ presentes nas flores de LAL e de 9,55\% nas flores de LAQ.

Os extratos de LAM e AGB, independentemente do solvente extrator, apresentam o mesmo perfil, sendo: a) incolor até $\mathrm{pH}$ acima de 10,01 para os extratos de AGB; b) incolor acima de $\mathrm{pH}$ 8,03 para os extratos de LAM; e c) amarelas após pH 11,02 para extratos de LAM e AGB. Esse comportamento provavelmente se deve ao baixo teor de antocianinas determinado no extrato das flores de LAM de $0,07 \%$ e de $0,21 \%$ no extrato das flores de AGB. Assim, os carotenoides presentes, especialmente $\beta$-caroteno e/ou luteína, ${ }^{49}$ promovem a visualização de soluções incolores ou amarelas. Esses carotenoides apresentam estabilidade frente às mudanças de $\mathrm{pH}$ do meio. ${ }^{36,58,59}$

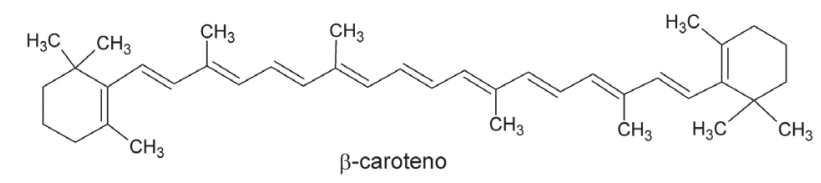<smiles>CC1=CC(O)CC(C)(C)C1/C=C/C(C)=C/C=C/C(C)=C/C=C/C=C(C)/C=C/C=C(C)/C=C/C1=C(C)CC(O)CC1(C)C</smiles>

Figura 11. Estruturas dos carotenoides $\beta$-caroteno e luteína ${ }^{32}$

Conforme Brouillard, ${ }^{11}$ soluções aquosas contendo antocianinas com valores de $\mathrm{pH}$ variando entre 1-14 podem resultar na produção de todas as cores do arco-íris. A partir das alterações visuais, fica claro que um grande número de reações deve estar envolvido no aparecimento, bem como no desaparecimento, de tantas cores. Isso é explicado pela presença das espécies $\mathrm{H}^{+}, \mathrm{OH}^{-}$e $\mathrm{H}_{2} \mathrm{O}$, devido à alta reatividade com as antocianinas, ${ }^{11}$ que são corantes naturais.

Dessa maneira, verifica-se que a extração das antocianinas responsáveis pela variação de $\mathrm{pH}$ observada nas tabelas colorimétricas, apresentadas nesta atividade experimental, ocorre de forma mais eficiente quando o meio é acidulado e na presença de calor. Também se observa que quanto mais próxima ao vermelho for a tonalidade das pétalas, maior a concentração de antocianinas e consequentemente mais interessante será a atividade aos estudantes, pois amplia o número de cores observadas com a variação do $\mathrm{pH}$ do meio.

$\mathrm{O}$ uso de extrato de plantas apresenta vantagens durante o processo de ensino e aprendizagem, ${ }^{60}$ pois quando a transmissão da informação acontece através dos canais verbal e visual a aprendizagem torna-se potencialmente mais efetiva ${ }^{61}$ Podemos destacar alguns pontos positivos desta atividade e do método proposto: i) valorização da grande variedade da flora, a qual varia de região para região, possibilitando a realização de uma atividade interdisciplinar com os estudantes; ii) o baixo custo para a execução da atividade; iii) as flores, utilizadas nesta atividade experimental, são recursos renováveis; iv) o processo de extração é simples. Esta atividade experimental ainda destaca a importância do uso de etanol acidulado, solvente clássico para a extração completa de antocianinas, ${ }^{16}$ permitindo também 
substituir indicadores clássicos de $\mathrm{pH}$ por indicadores alternativos de $\mathrm{pH}$ em realidades escolares que os indicadores clássicos de $\mathrm{pH}$ não estão disponíveis. Essas substituições contribuem para fortalecer os conceitos de química verde ${ }^{62}$ permitindo abordar conceitos de sustentabilidade e ambiente, minimizando a geração de resíduos tóxicos. Essa atividade experimental proposta contribui para aumentar o conhecimento sobre o comportamento de plantas de diferentes regiões do Brasil frente às variações de $\mathrm{pH}$ do meio, contribuindo para que independente da realidade escolar o professor possa abordar os conceitos de química envolvidos nessa atividade a partir de um corpo teórico que oriente a investigação proposta.

Ao final desta atividade experimental, propõe-se o mapa conceitual apresentado na Figura 12. Ele demonstra a integração dos conceitos das áreas de química, biologia, matemática e física. Choi e Park definem e destacam que ser multidisciplinar significa abordar o conhecimento de diferentes disciplinas mantendo-se dentro dos limites de cada uma. Já a interdisciplinaridade analisa, sintetiza e harmoniza tópicos de cada disciplina em um todo coordenado e coerente. ${ }^{63}$

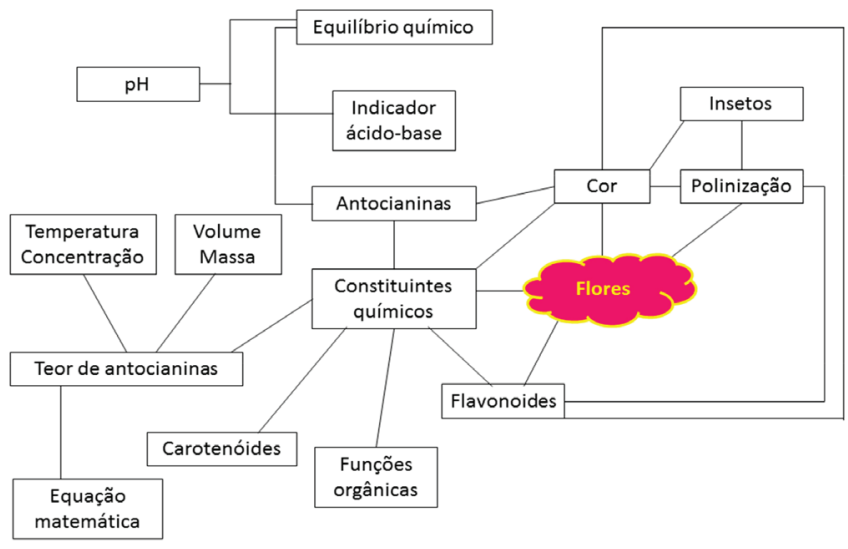

Figura 12. Mapa conceitual proposto partindo do tema flores

Após o desenvolvimento desta atividade experimental, ainda pode-se propor as seguintes questões para discussão com os estudantes:

1) Foi utilizado como solvente extrator: água, etanol e etanol acidulado com ácido clorídrico 1\%. Os resultados obtidos na tabela colorimétrica foram os mesmos? Justifique.

2) Discuta os conceitos ácido-base de Arrhenius, Brönsted-Lowry e Lewis frente aos equilíbrios químicos dos corantes apresentados nessa atividade.

3) O que justifica a variação mais intensa de cor nas tabelas colorimétricas a partir dos extratos de lírio amarelo queimado (LAQ)?

4) Você recomendaria testar que tipo de flores ou plantas para esse tipo de atividade?

5) Elabore um texto a partir de suas experiências cotidianas envolvendo o tema ácido-base.

6) Elabore um mapa conceitual a partir de suas concepções sobre o tema ácido-base.

\section{CONCLUSÃO}

Neste manuscrito propõe-se uma atividade experimental utilizando extratos de flores para abordar o tema ácido-base, sugerindo que dentre as flores cujo comportamento foi investigado os extratos recomendados como indicadores ácido-base alternativos sejam os extratos de lírio alaranjado (LAL) e amarelo queimado (LAQ). O alto teor de antociacinas determinados em LAL e em LAQ permitiu obter alterações significativas nas colorações das soluções entre pH 1-14. Além disso, a atividade contribui para a reflexão dos estudantes sobre "quais plantas posso usar?". A atividade deixa claro que plantas com elevado teor de antocianina são as mais próximas ao vermelho, sendo as concentrações determinadas em relação à cianidina-3-glicosídica em ordem crescente de 0,07 $( \pm 0,03)$ para $\mathrm{LAM}<0,21$ $( \pm 0,03)$ para $\mathrm{AGB}<1,72( \pm 0,13)$ para $\mathrm{AGV}<1,83( \pm 0,47)$ para $\mathrm{HAZ}<2,87( \pm 0,52)$ para LAL $<9,55( \pm 0,46)$ para LAQ. O melhor solvente para a extração é etanol/HCl $1 \%$, por provocar as maiores variações e intensidades de cor obtidas e, consequentemente, os melhores resultados experimentais do ponto de vista colorimétrico. Contudo, as flores investigadas LAL e LAQ apresentaram os maiores teores de antocianinas e as maiores alterações colorimétricas quando houve variação do $\mathrm{pH}$ da solução. Tendo esta atividade caráter inter e multidisciplinar, a construção de mapas conceituais torna-se uma estratégia interessante do ponto de vista pedagógico. Assim, a partir desta atividade experimental utilizando extratos de Lilium sp., Agapanthus sp. e Hydrangea sp. espera-se contribuir na elaboração ou na realização de atividades experimentais em cursos que demandam Química em sua matriz curricular.

\section{AGRADECIMENTOS}

Ao Laboratório de Corantes e Processos Pirolíticos (LACOPPI), ao Instituto Federal do Paraná (IFPR), Fundação Araucária (FA), Programa Institucional de Bolsas a Inclusão Social - Pesquisa e Extensão Universitária da Fundação Araucária (PIBIS-FA), Programa de Bolsas de Iniciação Científica da Fundação Araucária (PIBIC-FA), Conselho Nacional de Desenvolvimento Científico e Tecnológico (CNPq).

\section{REFERÊNCIAS}

1. Antunes, M.; Adamatti, D. S.; Pacheco, M. A. R.; Giovanela, M.; Quim. Nova Esc. 2009, 31, 283.

2. de Moraes, S. L.; Landgraf, M. D.; Rezende, M. O. O.; Ecletica Quim. 2004, 29, 47.

3. Previdello, B. A. F.; de Carvalho, F. R.; Tessaro, A. L.; de Souza, V. R.; Hioka, N.; Quim. Nova 2006, 29, 600.

4. Moreira, L. M.; de Moraes, P. C. G.; de Mendonça, J. P. R. F.; Guimarães, L.; Lyon, J. P.; Aimbire, F.; Poli, A. L.; Imasato, H.; Quim. Nova 2011, 34, 119.

5. de Ávila, S. G.; Matos, J.de R.; Educ. Quim. 2017, 28, 254.

6. Dangui, A. Z.; Santos, V. M. S.; Gomes, B. S.; de Castilho, T. S.; Nicolini, K. P.; Nicolini, J.; Spectrochim. Acta, Part A 2018, 203, 333.

7. Soares, M. H. F. B.; Cavalheiro, É. T. G.; Antunes, P. A.; Quim. Nova 2001, 24, 408 .

8. Raviolo, A.; Farré, A.; Educ. Quim. 2017, 28, 163.

9. Russel, J. B.; Química Geral - Volume 1, 2a ed., Pearson Makon Books: São Paulo, 1994.

10. Lee, J. D.; Química Inorgânica Não Tão Consisa, Edgard Blucher: São Paulo, 1999.

11. Brouillard, R.; Chapter 1 - Chemical Structure of Anthocyanins A2 Markakis, Pericles, Academic Press: Cambridge, Massachusetts, 1982.

12. Gürses, A.; Açıkyıldız, M.; Güneş, K.; Gürses, M. S.; Dyes and Pigments, Springer: New York, 2016, p. 13.

13. Castaneda-Ovando, A.; de Lourdes Pacheco-Hernández, M.; PáezHernández, M. E.; Rodríguez, J. A.; Galán-Vidal, C. A.; Food Chem. 2009, 113, 859 .

14. Silva, S.; Costa, E.; Calhau, C.; Morais, R.; Pintado, M.; Crit. Rev. Food Sci. Nutr. 2017, 57, 3072.

15. Timberlake, C. F.; Bridle, P. In Anthocyanins as Food Colors, Academic Press: Cambridge, Massachusetts, 1982, p. 125.

16. Harborne, J. B.; Phytochemical Methods: A Guide to Modern Techniques of Plant Analyses, $3^{\text {rd }}$ ed.: Chapman \& Hall: London, 1998, cap. 2. 
17. Shiono, M.; Matsugaki, N.; Takeda, K.; Nature 2005, 436, 791.

18. Mazza, G.; Brouillard, R.; Recent Developments in the Stabilization of Anthocyanins in Food Products 1987, 25, 207.

19. Stintzing, F. C.; Carle, R.; Int. J. Food Sci. Technol. 2004, 15, 19.

20. Fedenko, V. S.; Shemet, S. A.; Landi, M.; J. Plant Physiol. 2017, 212, 13.

21. Harborne, J. B.; Biochem. J. 1958, 70, 22.

22. Galloway, K. R.; Bretz, S. L.; Novak, M.; J. Chem. Educ. 2014, 92, 183.

23. Okumura, F.; Soares, M. H. F. B.; Cavalheiro, É. T. G.; Quim. Nova 2002, 25, 680 .

24. Couto, A. B.; Ramos, L. A.; Cavalheiro, E.; Quim. Nova 1998, 21, 221.

25. da Silva, D. B.; Gonçalves, M. de M.; Kreve, Y. D.; Nicolini, K. P.; Nicolini, J.; Educ. Quim. 2018, 29, 3.

26. Martins, R. de C.; Bernardi, F.; Kreve, Y. D.; Nicolini, K. P.; Nicolini, J.; Educ. Quim. 2017, 28, 246.

27. Soares, M. H. F. B.; Silva, M. V. B.; Cavalheiro, É. T. G.; Eclet. Quím. 2001, 26, 225.

28. Sonai, G. G.; Melo Jr., M. A.; Nunes, J. H. B.; Megiatto Jr., J. D.; Nogueira, A. F.; Quim. Nova 2015, 38, 1357.

29. Terci, D. B. L.; Rossi, A. V.; Quim. Nova 2002, 25, 684.

30. Fagundes, T. da S. F.; Pacheco, C. M.; Martins, P. R. C.; Valverde, A. L.; Ribeiro, C. M.; Rev. Virtual Quim. 2018, 10, 841.

31. Maestrin, A. P. J.; Neri, C. R.; Oliveira, K. T.d.; Serra, O. A.; Iamamoto, Y.; Quim Nova 2009, 32, 1670.

32. Bernardi, F.; Nicolini, K. P.; Nicolini, J.; Infarma 2017, 29, 68

33. Oliveira, M. A. B.; Pereira, S. T.; dos Anjos Mendonça, A. R.; de Souza Lopes, E. J.; Mundim, F. G. L.; Pereira, R. M.; Revista Eletrônica Acervo Saúde 2018, 11, e132.

34. Uma rápida busca ao google acadêmico feita em 16/08/2019 usandose "indicadores naturais de $p H$ " com o termo de busca revelou 65 resultados entre 2015-2019. Usando o termo de busca "natural pH indicator" ao Web of Science em 16/08/19 para os últimos 5 anos revelou 6 publicações sendo 4 provenientes de pesquisas realizados no Brasil.

35. Ribeiro, N. M.; Nunes, C. R.; Quim. Nova Esc. 2008, 29, 30.

36. Dias, M.; Guimarães, P.; Merçon, F.; Quim. Nova Esc. 2003, 17, 27.

37. da Costa, C. L. S.; Chaves, M. H.; Quim. Nova 2005, 28, 149.

38. Peralta, J.; Bitencourt-Cervi, C. M.; Maciel, V. B. V.; Yoshida, C. M. P.; Carvalho, R. A.; Food Packaging and Shelf Life 2019, 19, 47.

39. Prietto, L.; Pinto, V. Z.; El Halal, S. L. M.; de Morais, M. G.; Costa, J. A. V.; Lim, L.-T.; Dias, A. R. G.; Zavareze, E. de R.; J. Sci. Food Agric. 2018, 98, 2735.
40. Nogueira, S. A.; Sousa, L. R.; Silva, N. K. L.; Rodrigues, P. H. F.; Coltro, W. K. T.; Micromachines 2017, 8, 10.

41. Brilhante, S. E. T.; Valdevino, F.; de Oliveira Neto, F. B.; Silva, F. F. M.; Bertini, L. M.; Alves, L. A.; Holos 2015, 31, 3.

42. Sandre, A. A.; Pina, J. M.; Moraes, R. M.; Furlan, C. M.; Braz. J. Bot. 2014, 37, 9 .

43. Xu, R.; Ye, Y.; Zhao, W.; Introduction to Natural Products Chemistry, CRC Press - Taylor \& Francis Group: Boca Raton, 2012.

44. Scalzo, R. L.; Genna, A.; Branca, F.; Chedin, M.; Chassaigne, H.; Food Chem. 2008, 107, 136.

45. Yamagishi, M.; Sci. Hortic. 2013, 163, 27.

46. Xu, F.; Yuan, Q.; Dong, H.; J Chromatogr B 2006, 838, 44.

47. da Silveira, T. F. F.; Meinhart, A. D.; Coutinho, J. P.; de Souza, T. C. L.; Cunha, E. C. E.; de Moraes, M. R.; Godoy, H. T.; Food Res. Int. 2016, $82,165$.

48. Khan, P. M. A.; Farooqui, M.; J. Adv. Sci. Res. 2011, 2, 20.

49. Tanaka, Y.; Sasaki, N.; Ohmiya, A.; Plant J. 2008, 54, 733.

50. Cannon, M. B.; Ong, K. L. In Using Food to Stimulate Interest in the Chemistry Classroom; Symcox, K., ed.; American Chemical Society, 2013, p. 129.

51. Yamagishi, M.; Uchiyama, H.; Handa, T.; J. Plant Physiol. 2018, 228, 85.

52. Prietto, L.; Mirapalhete, T. C.; Pinto, V. Z.; Hoffmann, J. F.; Vanier, N. L.; Lim, L.-T.; Guerra Dias, A. R.; da Rosa Zavareze, E.; Food Sci. Technol. 2017, 80, 492

53. Mori, S.; Otani, M.; Kobayashi, H.; Nakano, M.; Sci. Hortic. 2014, 166, 24.

54. Miyahara, T.; Takahashi, M.; Ozeki, Y.; Sasaki, N.; J. Plant Physiol. 2012, 169, 1321.

55. Bloor, S. J.; Falshaw, R.; Phytochemistry 2000, 53, 575.

56. Yoshida, K.; Toyama-Kato, Y.; Kameda, K.; Kondo, T.; Plant Cell Physiol. 2003, 44, 262.

57. Yoshida, K.; Ito, D.; Shinkai, Y.; Kondo, T.; Phytochem 2008, 69, 3159.

58. Courraud, J.; Berger, J.; Cristol, J.-P.; Avallone, S.; Food Chem. 2013, 136,871 .

59. Rizk, E. M.; El-Kady, A. T.; El-Bialy, A. R.; Ann. Agric. Sci. 2014, 59, 53.

60. Hartwell, S. K.; Chem. Educ. Res. Pract. 2012, 13, 135.

61. Tavares, R.; Ciências \& Cognição 2008, 13, 94.

62. Gonçalves, F. P.; Yunes, S. F.; Guaita, R. I.; Marques, C. A.; Pires, T. C. M.; Pinto, J. R. M.; Machado, A. A. S. C.; Educ. Quim. 2017, $28,99$.

63. Choi, B. C.; Pak, A. W.; Clin. Invest. Med. 2006, 29, 351. 